OPEN ACCESS

Edited by:

Haichang Li,

The Ohio State University,

United States

Reviewed by:

Taeg Kyu Kwon,

Keimyung University Dongsan Medical

Center, South Korea

Jin Won Hyun,

Jeju National University, South Korea

Dong-Hua Yang,

St. John's University, United States

*Correspondence:

Cheng-yun Jin

cyjin@zzu.edu.cn

Hai-wei Xu

Xhwei01@126.com

Zhengya Wang

zhenyawang@zzu.edu.cn

${ }^{\dagger}$ These authors have contributed equally to this work and share first authorship

Specialty section:

This article was submitted to

Pharmacology of Anti-Cancer Drugs,

a section of the journal

Frontiers in Oncology

Received: 26 January 2021

Accepted: 22 March 2021

Published: 16 April 2021

Citation:

Wang $M$, Wu X, Yu L, Hu Z-y,

Li X, Meng X, Lv C-t, Kim G-Y,

Choi $Y H$, Wang Z, Xu H-W and Jin C-Y (2021) LCT-3d Induces Oxidative

Stress-Mediated Apoptosis by

Upregulating Death Receptor 5

in Gastric Cancer Cells.

Front. Oncol. 11:658608.

doi: 10.3389/fonc.2021.658608

\section{LCT-3d Induces Oxidative Stress- Mediated Apoptosis by Upregulating Death Receptor 5 in Gastric Cancer Cells}

\author{
Menglin Wang ${ }^{1 \dagger}$, Xinxin $\mathrm{Wu}^{1+}$, $\mathrm{Lu} \mathrm{Yu}^{1 \dagger}$, Zi-yun $\mathrm{Hu}^{1 \dagger}$, Xiaobo $\mathrm{Li}^{1}$, Xia Meng ${ }^{1}$, \\ Chun-tao Lv ${ }^{1}$, Gi-Young Kim ${ }^{2}$, Yung Hyun Choi ${ }^{3}$, Zhengya Wang ${ }^{1 *}$, Hai-Wei Xu ${ }^{1 *}$ \\ and Cheng-Yun Jin ${ }^{1,4 *}$

\begin{abstract}
${ }^{1}$ Key Laboratory of Advanced Technology for Drug Preparation, Ministry of Education, School of Pharmaceutical Sciences, Zhengzhou University, Zhengzhou, China, ${ }^{2}$ Department of Marine Life Sciences, Jeju National University, Jeju, South Korea, ${ }^{3}$ Department of Biochemistry, College of Oriental Medicine, Dong-Eui University, Busan, South Korea, ${ }^{4}$ State Key
\end{abstract} \\ Laboratory of Esophageal Cancer Prevention \& Treatment, Zhengzhou University, Zhengzhou, China
}

Gastric cancer is a global health problem. In this study, we investigate the role of a novel Indole derivative, named LCT-3d, in inhibiting the growth of gastric cancer cells by MTT assay. The Western blotting results showed that LCT-3d modulated the mitochondrialrelated proteins and Cleaved-Caspases 3/9, to induce cell apoptosis. The up-regulation of Death receptor 5 (DR5) in MGC803 cells was observed with LCT-3d treatment. Knockdown of DR5 on MGC803 cells partially reversed the LCT-3d-induced mitochondrial apoptosis. The level of Reactive Oxygen Species (ROS) in MGC803 cells was increased with LCT-3d treatment and could be blocked with the pretreatment of the ROS inhibitor N-Acetylcysteine (NAC). The results demonstrate that the elevating ROS can up-regulate the expression of DR5, resulting in apoptosis via mitochondrial pathway. Although the nuclear factor erythroid-2 related factor 2 (Nrf2) pathway served an important role in protecting gastric cancer cells against the injury of ROS, it can't reverse LCT-3dinduced cell apoptosis. Taken together, our study showed that LCT-3d induced apoptosis via DR5-mediated mitochondrial apoptotic pathway in gastric cancer cells. LCT-3d could be a novel lead compound for development of anti-cancer activity in gastric cancer.

Keywords: LCT-3d, DR5, reactive oxygen species, Nrf2, apoptosis, gastric cancer

\section{INTRODUCTION}

Gastric cancer remains the third leading cause of death in spite of the decreasing incidence and mortality (1). Many people in China are diagnosed with gastric cancer every year, and once diagnosed, most of them are in the advanced stage with metastasis (2). Currently, chemotherapy for gastric cancer is limited due to serious side effects and low efficacy (3). The development of drug resistance in tumors is a major obstacle of chemotherapy (4-7). Hence, developing novel effective strategies for treatment of gastric cancers is warranted. 
Drugs with Indole skeleton shows good efficacy for cancer therapy (8), such as vincristine (9), Indole-3-carbinol (I3C) (10), BPR0L075 (11), as well as JKA97 (12). All of them have exhibited good effects on anti-tumor through inducing cell apoptosis via a variety of signaling pathways.

In the previous work, we synthesized a potent Lysine (K)Specific Demethylase 1A (LSD1) inhibitor, named LCT-9e (Figure 1B), which effectively inhibits the macrophages (THP1 cells) growth (13). LCT-9e is the first irreversible LSD1 inhibitor, which is not derived from monoamine oxidase inhibitors. Hence, LCT-9e was chosen to be a lead compound for optimization. Unexpectedly, when the heterocyclic group was introduced to C-5 position of the Indole, the LSD1 inhibitors such as compound LCT-3d kept the anti-proliferation activity in gastric cancer cells with low cytotoxicity. In this study, we explored the role of LCT-3d in gastric cancer cells and its molecular mechanism. The results suggested that LCT-3d induced the generation of Reactive Oxygen Species (ROS), which subsequently led to gastric cancer cell apoptosis via upregulating the expression of Death receptor 5 (DR5). Meanwhile, LCT-3d activated the nuclear factor erythroid-2 related factor 2 (Nrf2) pathway, which attenuated LCT-3d-induced cell apoptosis. This is the first report on Indole derivative in inducing apoptosis in gastric cancer cells through DR5mediated pathway.

\section{MATERIALS AND METHODS}

\section{Reagents and Antibodies}

LCT-3d was synthesized in our group and was dissolved in DMSO. RPMI-1640, Fetal bovine serum (FBS), and penicillinstreptomycin were purchased from HyClone (Victoria, Australia). DCFH-DA (2, 7-dichlorodihydrofuorescein diacetate), the Nuclear and Cytoplasmic Protein Extraction Kit, and Annexin V- FITC Apoptosis Detection Kit were purchased from Beyotime Biotechnology (Shanghai, China). The primary antibodies for Caspase 3 (sc-7272), PARP (sc-7150), DR5 (sc65314), Caspase 9 (sc-7885), Bim (sc-11425), Bad (sc-8044), Bax (sc-493), DR4 (sc-7863), and $\beta$-actin (sc-1615) were purchased from Santa Cruz Biotechnology (Santa Cruz, CA, USA). Bid (\#2002), Bcl-xL (\#2764), and XIAP (\#14334) were purchased from Cell Signaling Technology (Danvers, MA, USA). The secondary antibodies anti-goat, anti-rabbit, and anti-mouse were purchased from Bioss (Shanghai, China). The panCaspase inhibitor Z-VAD-FMK was purchased from Selleck. The Nrf2 inhibitor ML385 was purchased from MCE (NJ, USA). The ECL (enhanced chemiluminescence) kit was purchased from Thermo Fisher (Waltham, MA, USA). Anti-DR5 (ab1675) antibody for flow cytometry was purchased from Abcam (Cambridge, MA, USA). NAC (N-acetyl-L-cysteine), MTT [3(4, 5-dimethylthiazol-2-yl) - 2, 5-diphenyltetra- zolium

A

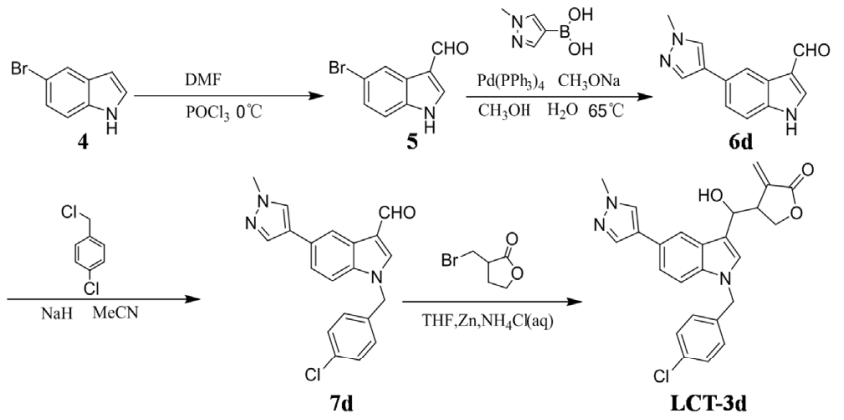

B

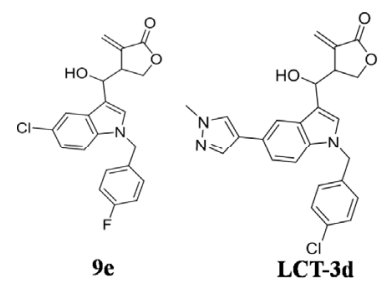

C

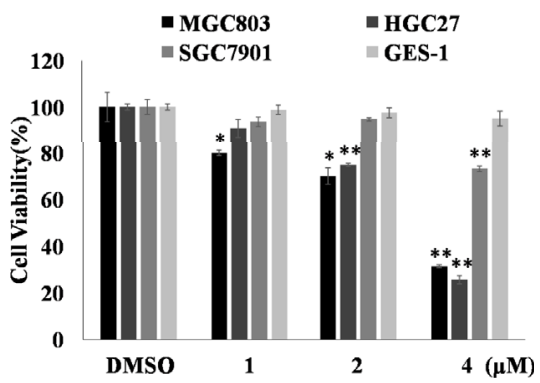

FIGURE 1 | LCT-3d inhibited the cell proliferation of gastric cancer cells. (A) LCT-3d synthetic procedure. (B) Structure of LCT-3d. (C) Cytotoxic effect of LCT-3d on gastric cancer cells measured by MTT assay. Cells were treated with increasing concentration of LCT-3d for $48 \mathrm{~h}$. ${ }^{*} P<0.05$, ${ }^{* *} P<0.01$, significantly different compared with control. 
bromide], and JC-1 fluorescent dye were purchased from SigmaAldrich (St. Louis, MO, USA).

\section{Cell Lines and Culture}

Gastric cancer cells MGC803, HGC27, SGC7901 and normal gastric cells GES-1 were from the American Type Culture Collection. These cells were cultured in cell medium supplemented with $10 \% \mathrm{FBS}$ in an incubator with $5 \% \mathrm{CO}_{2}$ at $37^{\circ} \mathrm{C}$. The DR5 knockout cell line of MGC803 was previously constructed by our team (14).

\section{Cell Viability Assay}

The viability of gastric cancer cells and GES-1 cells were examined by MTT assay. After cells were incubated with LCT-3d for 48 h, 20 $\mu \mathrm{l}$ MTT $(5 \mathrm{mg} / \mathrm{ml})$ was added into each well. Subsequently, cells were incubated for another $2-4 \mathrm{~h}$, the cell culture medium was removed, and then $150 \mu \mathrm{l}$ DMSO was added into each well. Optical density for MTT assay was detected at $490 \mathrm{~nm}$ with a microplate reader (Synergy H1, BioTek, VT, USA). The dose of DMSO has no effect on cell viability in all groups of cells in this study.

\section{Apoptosis Analysis}

MGC803 and HGC27 cells were treated with LCT-3d at different concentrations. After incubating for $48 \mathrm{~h}$, the cells were collected and stained with Annexin V and PI for $20 \mathrm{~min}$. Next, cells were measured by a flow cytometer (BD LSRFortessa ${ }^{\mathrm{TM}}$ Cell Analyzer, Becton, Dickinson and Company, NJ, USA). The data was quantified by the FlowJo software.

\section{Measurement of Mitochondrial Membrane Potential (MMP, $\Delta \Psi$ )}

MGC803 and HGC27 cells were treated with LCT-3d at different concentrations. After incubating for $48 \mathrm{~h}$, the cells were collected and then incubated with JC-1 $(2.5 \mu \mathrm{g} / \mathrm{ml})$ for $10 \mathrm{~min}$ at $37^{\circ} \mathrm{C}$. Next, cells were measured by a flow cytometer. The data was quantified by the FlowJo software (15).

\section{Analysis of DR5 Expression by a Flow Cytometer}

Wild type MGC803 and DR5 ${ }^{-1-}$ MGC803 cells were seeded in a six-well plate and treated with LCT-3d for $24 \mathrm{~h}$. The cells were collected and fixed by $1 \%$ paraformaldehyde at $4^{\circ} \mathrm{C}$ for $30 \mathrm{~min}$. Then the cells were incubated with normal goat serum, DR5 antibody, and Goat anti-mouse IgG successively. Next, the cells were measured by a flow cytometer. The data was quantified by FlowJo software.

\section{Measurement of ROS}

MGC803 and HGC27 cells were treated with LCT-3d for indicated time. Then cells were collected and stained with DCFH-DA for $20 \mathrm{~min}$. Next, the cells were measured by a flow cytometer. The data was quantified by FlowJo software.

\section{Total/Nuclear Protein Extraction}

MGC803 cells were treated with LCT-3d for indicated time. Then the cells were collected and proteins were extracted according to instruction of the Nuclear and Cytoplasmic
Protein Extraction Kit. The protein concentration was determined by the micro-BCA protein assay kit.

\section{Western Blotting}

MGC803 and HGC27 cells were treated with agents for indicated time. The total proteins were collected and quantified by the micro-BCA protein assay kit (P0012, Beyotime, Shanghai, China). Then the proteins were subjected to immunoblotting as previously described (16).

\section{Immunofluorescence}

Cells were treated with agents for indicated time, then immunofluorescence analysis was performed as reported previously (17).

\section{Statistical Analysis}

All experiments were repeated at least three times. The data were presented as mean \pm SD. Differences between the experimental groups were determined by paired or unpaired Student's t test, ${ }^{*}$ and ${ }^{* *}$ represent $P<0.05$ and $P<0.01$, respectively.

\section{RESULTS}

\section{LCT-3d Reduced the Cell Viability of Human Gastric Cancer Cells but Not Normal Cells}

The process of synthesize LCT-3d as shown in Figure 1A. The effect of LCT-3d on three human gastric cancer cells (MGC803, HGC27, SGC7901) and normal cell GES-1 were evaluated by MTT assay. The results showed that the viability of MGC803 and HGC27 cells were reduced in a dose-dependent manner after treatment with LCT-3d (Figure 1C). In addition, MGC803 and HGC27 cells exhibited more sensitivity to LCT-3d than SGC7901 cells, with the inhibitory rate of 68.83 and $74.41 \%$ at $4 \mu \mathrm{M}$, respectively. LCT-3d showed little inhibition on the viability of GES-1 cells after treatment with LCT-3d. These results demonstrated that LCT-3d decreased the growth of human gastric cancer cells but not normal cells.

\section{LCT-3d Induced Cell Apoptosis Dependent on Caspases}

In order to determine whether LCT-3d induced cell apoptosis, the apoptotic rate of MGC803 and HGC27 cells was detected by flow cytometry. The apoptotic rate was increased in a concentration-dependent manner in both cells (Figure 2A). Then some Caspase-related proteins were tested (Figure 2B). Western blotting results demonstrated that LCT-3d promoted the expression of Cleaved-PARP and Cleaved-Caspase 3/9 in MGC803 and HGC27 cells.

To further confirm whether Caspase family proteins regulated LCT-3d-induced apoptosis, the cells were pretreated with a panCaspase inhibitor Z-VAD-FMK $(100 \mu \mathrm{M})$ for $1 \mathrm{~h}$, and subsequently incubated with LCT-3d $(4 \mu \mathrm{M})$ for an additional $48 \mathrm{~h}$. The flow cytometry analysis showed that pretreatment with Z-VAD-FMK $(100 \mu \mathrm{M})$ partially reversed LCT-3d-induced 
A

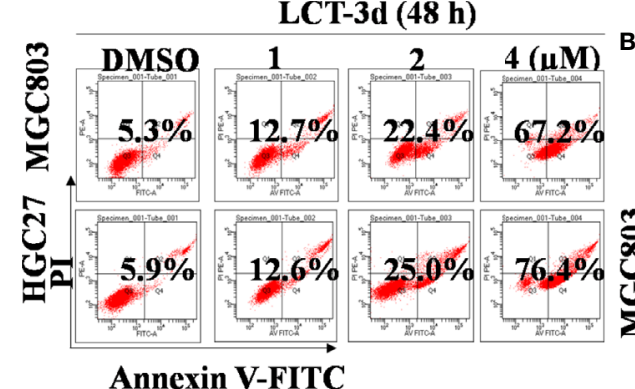

LCT-3d (48 h)

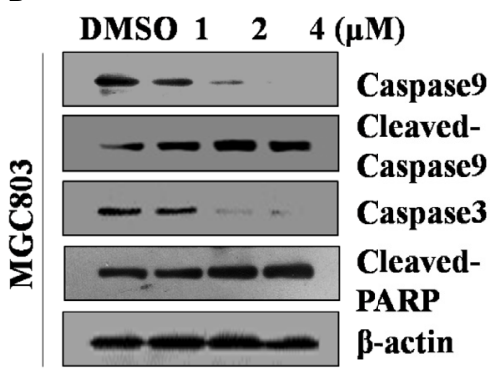

C

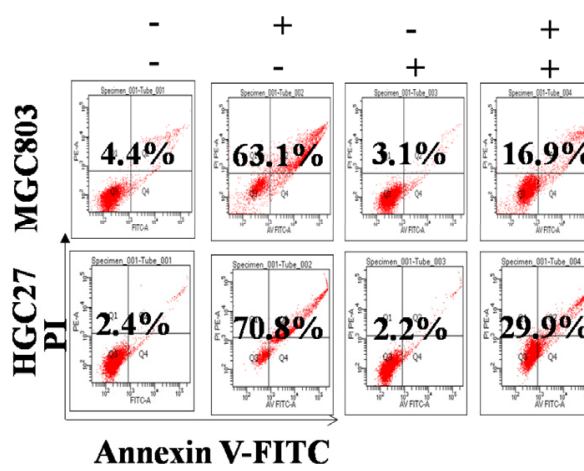

LCT-3d (48 h)

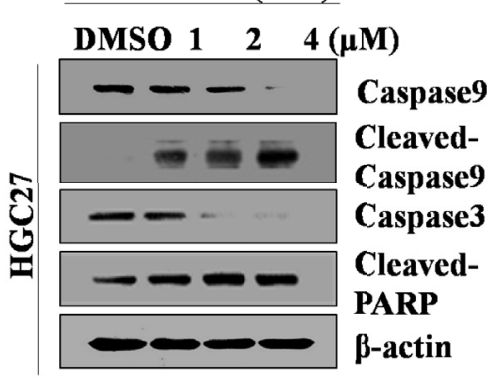

FIGURE 2 | LCT-3d triggered Caspase mediated apoptotic pathway in gastric cancer cells. (A) MGC803 cells and HGC27 were cells treated with various concentrations of LCT-3d for $48 \mathrm{~h}$ and apoptosis analyzed by flow cytometry. (B) Cells were treated as in (A) and the expression of Cleaved-Caspase and Cleaved PARP was analyzed by Western blotting. (C) MGC803 cells and HGC27 cells were pretreated with a pan-Caspase inhibitor, Z-VAD-FMK (100 $\mu$ M) for 1 h, followed by incubation with LCT-3d (4 $\mu \mathrm{M})$ for $48 \mathrm{~h}$. Flow cytometric analysis on the effect of Z-VAD-FMK on LCT-3d-induced cells apoptosis.

apoptosis in MGC803 and HGC27 cells (Figure 2C). These results suggested that LCT-3d induced apoptosis of the gastric cancer cells through a Caspase-dependent manner.

\section{LCT-3d Regulated the Expression of Bcl-2 Family Proteins and Reduced the Mitochondrial Membrane Potential (MMP/ $\Delta \Psi)$}

To further explore the underlying mechanism of LCT-3dinduced apoptosis, the change of mitochondrial membrane potential $(\Delta \Psi)$ and the level of proteins involved in apoptosis were investigated. It was found that LCT-3d reduced the mitochondrial membrane potential in MGC803 and HGC27 cells (Figure 3A). Furthermore, LCT-3d down-regulated the expression of anti-apoptotic proteins $\mathrm{Bcl}-2, \mathrm{Bcl}-\mathrm{xl}, \mathrm{Bid}$, and XIAP, and up-regulated the pro-apoptotic proteins Bax, Bad, and Bim in MGC803 cells (Figure 3B). These results demonstrated that LCT-3d induced apoptosis of gastric cancer cells via the mitochondrial pathway.

\section{DR5 Was Involved in LCT-3d-Induced Apoptosis in MGC803 Cells}

Then, the role of DR5 was evaluated on LCT-3d-induced apoptosis. It was found that LCT-3d significantly up-regulated the expression of DR5 in MGC803 cells (Figure 4A). To further confirm whether DR5 is necessary for LCT-3d-induced apoptosis, the DR5 knocked down MGC803 cells were used for investigation. Flow cytometry analysis showed that the expression of DR5 was reduced in DR5 knocked down MGC803 cells (Figure 4B). The LCT-3d-induced cell death was partially reversed in DR5 knocked down MGC803 cells (Figures 4C, D). The Cleaved-Caspase 9 and Cleaved-PARP were decreased in DR5 knocked down MGC803 cells compared with wild type MGC803 cells (Figure 4E). LCT-3d-induced mitochondrial depolarization was partially reversed in DR5 knocked down MGC803 cells (Figure 4F). Collectively, these results suggested that LCT-3d-induced apoptosis was mediated by DR5.

\section{LCT-3d Induced ROS Production and Activated Nrf2 Pathway in MGC803 Cells}

To explore whether ROS was related to LCT-3d-induced apoptosis, the levels of ROS were detected after treatment with LCT-3d by flow cytometry. The results showed that the level of ROS was markedly increased in a time-dependent manner in MGC803 and HGC27 cells (Figure 5A). Next, we detected the role of Nrf2 in LCT-3d-induced ROS generation in MGC803 cells. The results showed that LCT-3d up-regulated p-Nrf2 and its downstream targets $\mathrm{HO}-1$ and $\mathrm{NQO} 1$ in MGC803 cells (Figure 5B). Moreover, levels of Nrf2 in the nuclear fraction 
A

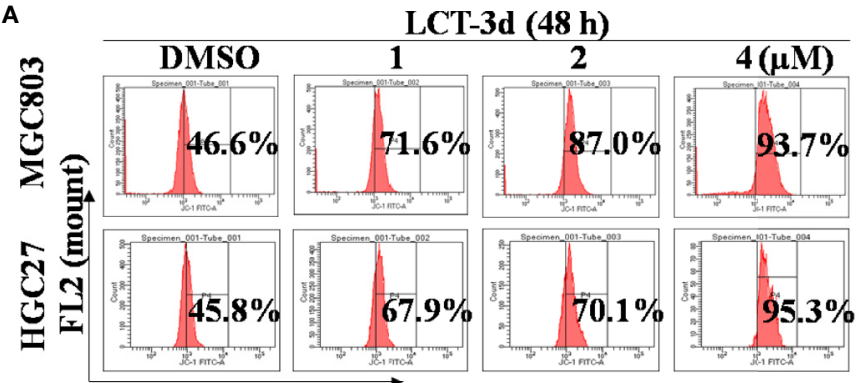

FL1 (JC-1 monomers)

B

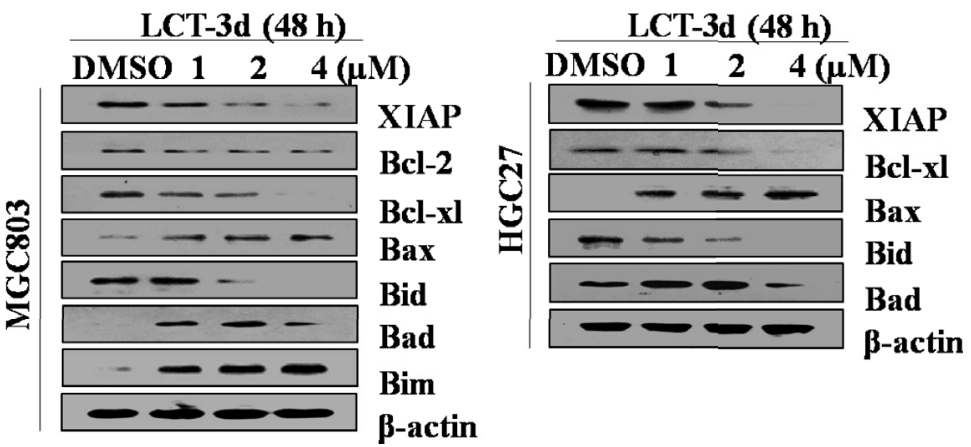

FIGURE 3 | LCT-3d-induced apoptosis was associated with mitochondrial pathway in gastric cancer cells. (A) MGC803 cells and HGC27 cells were treated with various concentrations of LCT-3d for $48 \mathrm{~h}$ and the membrane potential was measured by JC-1 dye retention using flow cytometry. (B) Cells were treated as in (A) and the expression of Bax, Bad, Bim, Bid, Bcl-xL, Bcl-2, and XIAP proteins was determined by Western blotting.

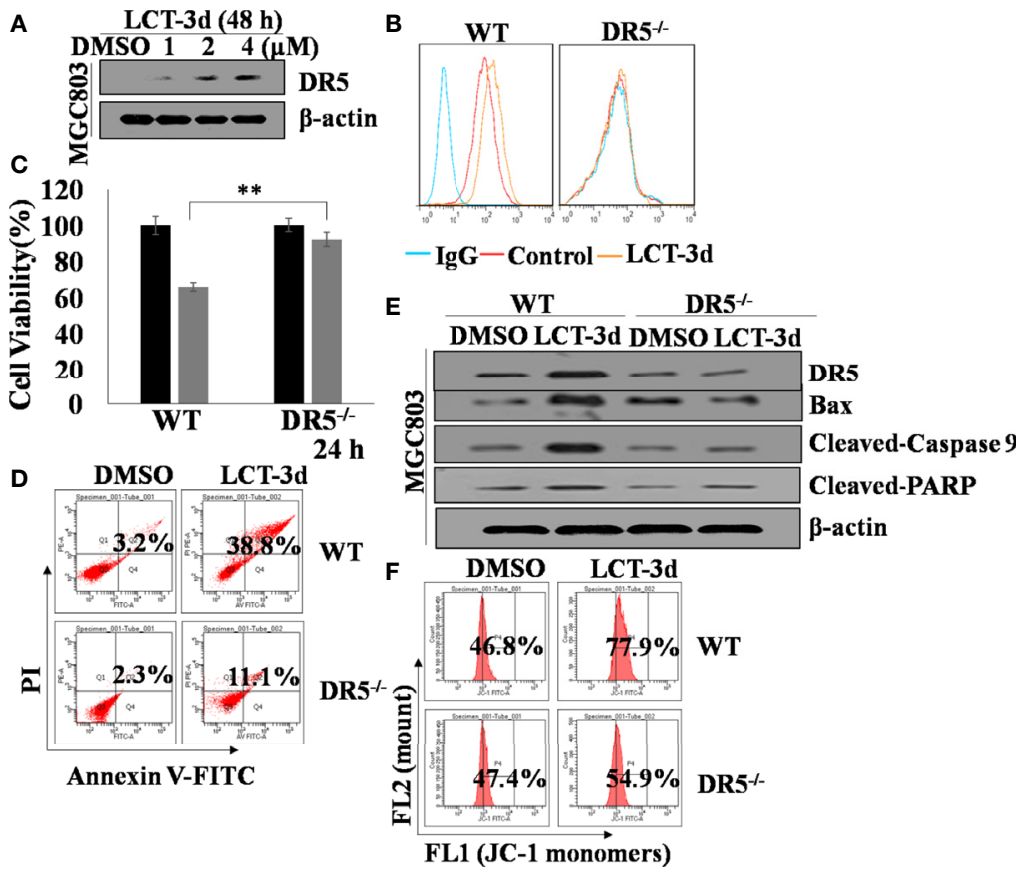

FIGURE 4 | LCT-3d-induced apoptosis is associated with extrinsic pathway in gastric cancer cells. Both wild type and DR5 ${ }^{-/-}$MGC803 cells were treated with 4 $\mu \mathrm{M}$ LCT-3d or indicated concentration. (A) Western blotting assay showed reduced expression of DR5 in a dose-dependent manner in MGC803 cells. (B) MGC803 cells and DR5 ${ }^{-/-}$MGC803 cells were treated with $4 \mu \mathrm{M}$ LCT-3d for 24 h and DR5 protein expressions in MGC803 cells determined by flow cytometry. (C) MTT assay showed the cell viability after treatment with $4 \mu \mathrm{M} \mathrm{LCT}-3 \mathrm{~d}$ for $24 \mathrm{~h}$. ${ }^{* *} \mathrm{P}<0.01$, significantly different compared with control. (D) Flow cytometric analysis showed the ratios of apoptotic cells after treatment with $4 \mu \mathrm{M}$ LCT-3d for $24 \mathrm{~h}$. (E) Western blotting assay showed the expression of the apoptosis-related proteins after treatment with $4 \mu \mathrm{M} L C T-3 d$ for $24 \mathrm{~h}$. (F) Flow cytometric analysis demonstrated the reduction of MMP $(\Delta \Psi)$ after treatment with $4 \mu \mathrm{M} L \mathrm{CT}-3 \mathrm{~d}$ for $24 \mathrm{~h}$. 

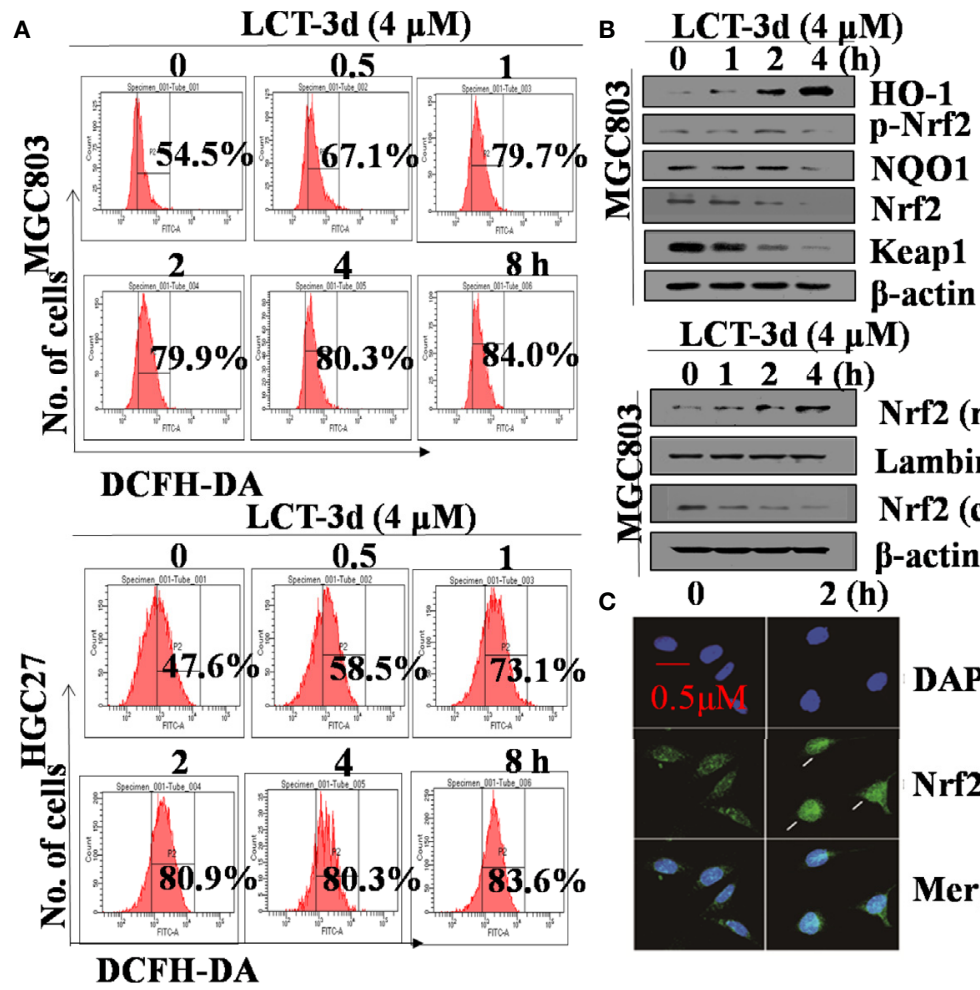

LCT-3d $(4 \mu \mathrm{M})$

$\begin{array}{lllll} & 1 & 2 & 4(\mathrm{~h})\end{array}$

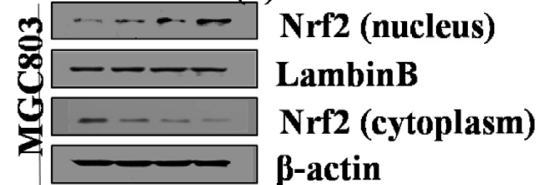

C
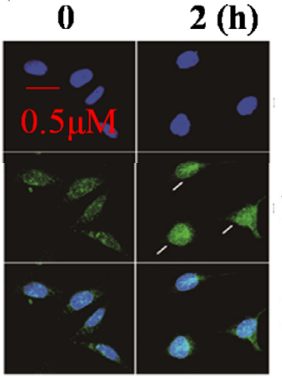

DAPI

Nrf2-FITC

Merge

FIGURE 5 | LCT-3d increased the level of ROS in gastric cancer cells. (A) MGC803 cells and HGC27 cells were treated with LCT-3d (4 $\mu$ M) for indicated times and the level of ROS was detected by DCFH-DA with flow cytometry. (B) Western blotting assay showed Nrf2 nuclear-translocation and the changes of LCT-3d-induced protein expression at indicated time points. (C) MGC803 cells were treated with LCT-3d (4 $\mu$ M) for $2 \mathrm{~h}$. The treated and untreated samples are stained with Nrf2 antibody (Green) and DAPI (Blue) (magnification, 400x). The arrows indicate Nrf2 nuclear translocation.

were significantly increased, whereas levels of Nrf2 in the cytoplasm were decreased, indicating that Nrf2 translocated into the nucleus and activated its downstream target genes (Figure 5B). To support this point, the immunofluorescence assay was conducted and similar result was observed (Figure 5C). These results suggested that LCT-3d induced the production of ROS and triggered the Nrf2 pathway to inhibit apoptosis of gastric cancer cells.

\section{LCT-3d-Induced Apoptosis Depends on ROS but Not Nrf2 in Gastric Cancer Cells}

To explore whether ROS contributed to the apoptosis induction, MGC803 and HGC27 cells were pretreated with NAC for $1 \mathrm{~h}$ followed by the treatment with LCT-3d $(4 \mu \mathrm{M})$ for an additional $48 \mathrm{~h}$. The pretreatment with NAC reversed the cell death in MGC803 and HGC27 cells (Figures 6A, B). The effect of ROS on cell apoptosis-related proteins was investigated by Western blotting. The results showed that NAC markedly reversed the changes of expression of apoptosis-related proteins, as well as abrogated mitochondrial depolarization. These results were similar in HGC27 cells to MGC803 cells (Figures 6C, D). However, the pretreatment with ML385 rarely reversed the cell death in MGC803 cells (Figure 6E). These results suggested that
LCT-3d-induced apoptosis depended on ROS but not Nrf2 in gastric cancer cells.

\section{DISCUSSION}

The death rate of patients suffering from gastric cancer is increasing. The emergence of side effects and multi-drug resistance to chemotherapeutic drugs usually lead to the failure of chemotherapies (18). In this study, a new Indole compound LCT-3d was discovered, which had selective cytotoxicity against gastric cancer cells with no apparent cytotoxicity against nonmalignant gastric epithelial cells. ROS level was detected by flow cytometry in gastric cancer cells. The cells pretreated with NAC for $1 \mathrm{~h}$ blocked the production of ROS and prevented the upregulation of DR5, as well as mitochondria JNK-CHOP pathway (18). ROS triggered apoptosis by activating death receptors and the mitochondria pathway $(19,20)$. Mechanistically, the data demonstrated that LCT-3d induced gastric cell apoptosis through DR5-mediated mitochondrial pathway.

The activation of Caspase family proteins is an important event leading to cell apoptosis (21). At the early stage of apoptosis, Caspase 3 and Caspase 9 were activated, and they served as biomarker proteins of apoptosis (22). Caspase 3, 
A

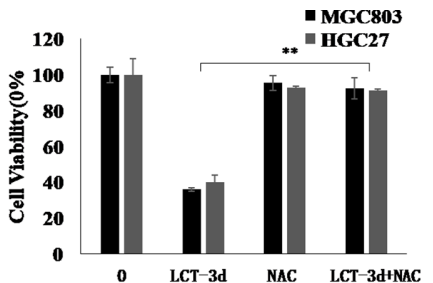

C
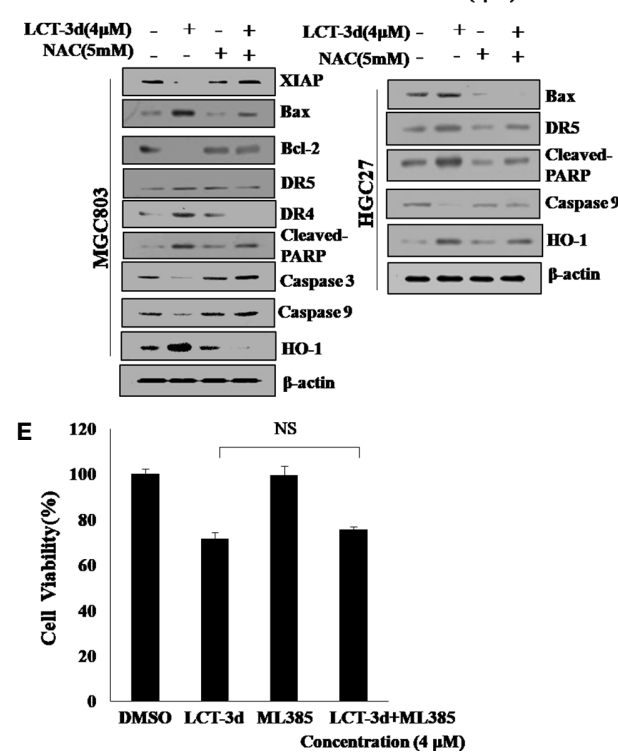

B

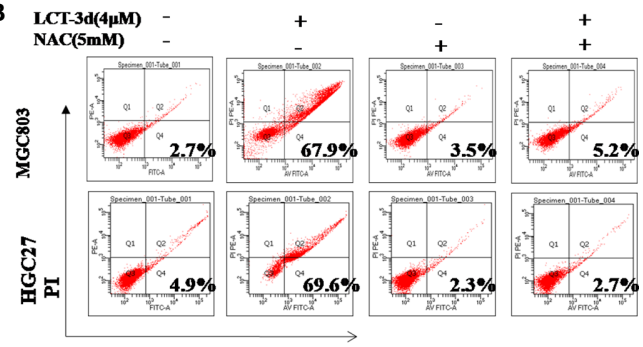

Annexin V-FITC

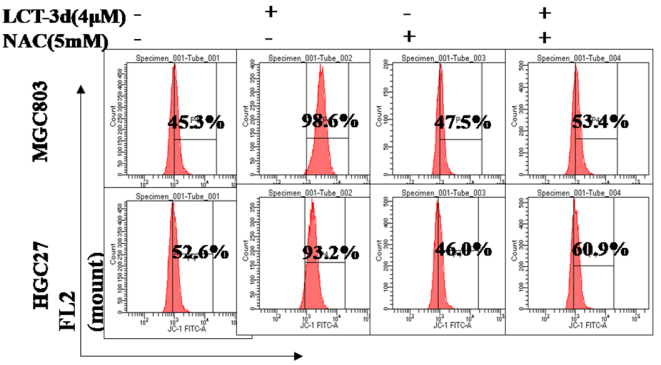

FL1 (JC-1 monomers)

FIGURE 6 | LCT-3d-induced apoptosis is associated with the rising ROS in gastric cancer cells. (A) MGC803 cells and HGC27 cells were pretreated with NAC $(5 \mathrm{mM})$ for $1 \mathrm{~h}$, followed by incubation with LCT-3d $(4 \mu \mathrm{M})$ for $48 \mathrm{~h}$. The effect of NAC on LCT-3d-induced cell death was analyzed by MTT assay. ${ }^{* *} P<0.01$, significantly different compared with control. (B) Flow cytometric analysis demonstrated the effects of NAC (5 mM) on LCT-3d (4 $\mu$ M)-induced cell apoptosis of the gastric cancer cells. (C) Western blotting assay showed the effect of NAC (5 mM) on LCT-3d (4 $\mu$ M)-induced change of protein expression at $48 \mathrm{~h}$. (D) Flow cytometric analysis showed the effect of NAC $(5 \mathrm{mM})$ on LCT-3d $(4 \mu \mathrm{M})$-induced loss of MMP $(\Delta \Psi)$ in gastric cancer cells. (E) MGC803 cells pretreated with ML385 for $1 \mathrm{~h}$ followed by the treatment with LCT-3d $(4 \mu \mathrm{M})$ for an additional $48 \mathrm{~h}$. The pretreatment with ML385 rarely reversed the cell death in MGC803 and HGC27 cells.

the key execution enzyme, was activated by Caspase 9, which is the primary initiator Caspase (23). The cleavage of PARP was regarded as an important indicator of apoptosis (24). Therefore, the expression of these proteins was measured by Western blotting. The results showed the activation of Cleaved-Caspase $3 / 9$ and Cleaved-PARP after treatment with LCT-3d. The reversion of the process by Z-VAD-FMK confirmed that LCT$3 \mathrm{~d}$ induced apoptosis dependent on Caspases.

It has long been reported that mitochondria is an important factor in the process of apoptosis (25). To further confirm whether LCT-3d treatment activated mitochondrial pathway, we detected mitochondria-related proteins in gastric cancer cells. Bcl-2 family proteins are the major regulators and effectors of the mitochondrial apoptotic pathway, which include anti-apoptotic proteins such as Bcl-2 and pro-apoptotic proteins such as Bax (26-29). The oligomerization of Bax and Bak proteins promote the increase of the mitochondrial membrane permeability, which leading to the release of cytochrome c into the cytosol, subsequently cell apoptosis occurred through Caspase cascade. In this work, mitochondrial membrane potential and the levels of $\mathrm{Bcl}-2$ and $\mathrm{Bcl}-\mathrm{xL}$ were decreased upon LCT-3d treatment. Meanwhile, pro-apoptotic protein Bax, Bad, and Bim were increased after LCT-3d treatment. Collectively, the findings demonstrated that LCT-3d induced apoptosis of gastric cancer cells via mitochondrial pathway.

The role of DR5 in LCT-3d-induced apoptosis was investigated. MTT assay showed that LCT-3d-induced cell death was partially reversed in MGC803-DR5 ${ }^{-/-}$cells, suggesting that DR5 is an important factor for inducing cell apoptosis upon LCT-3d treatment. Moreover, compared with wild type MGC803 cells, the apoptotic rate and the decline of MMP as well as the cleavage of PARP and Caspase 9 were reversed in MGC803-DR5 $5^{-/}$cells. These results demonstrated that activation of mitochondrial pathway was associated with DR5.

ROS can trigger the mitochondria-mediated intrinsic apoptotic pathway $(19,30)$. In this work, the elevating ROS in the process of LCT-3d-induced apoptosis was observed. In addition, NAC markedly inhibited the activation of Caspase 3/9, the upregulation of DR5, the modulation of Bcl-2 family proteins, as well as the cancellation of MMP decrease. These results suggested that NAC blocked the effect of ROS on gastric cancer cells, 


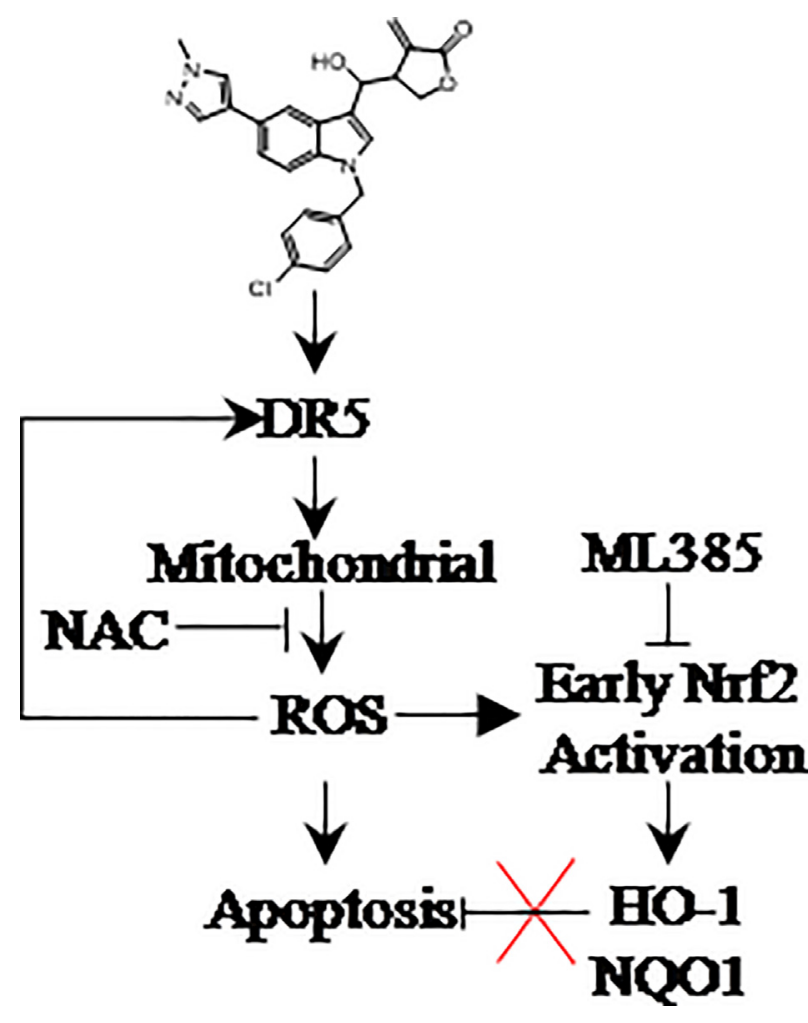

FIGURE 7 | LCT-3d selectively kills gastric cancer cells but not normal cells via inducing ROS triggered apoptosis by activating death receptors and the mitochondria pathway. ROS scavenger NAC can block cell death, while Nrf2 inhibitor ML385 has no effect on LCT-3d-induced cell death. The promotion of ROS-induced apoptosis by LCT-3d depends on the up-regulation of DR5 but not the inactivation of Nrf2.

indicating that ROS triggered cell death through DR5-mediated mitochondrial apoptosis pathway (31).

ROS are the key molecules in the process of apoptosis (32, 33). It is reported that moderate level of ROS can increase the ability of cell survival (34). It is well known that Nrf2 can protect cells from injury by oxidative stress through separating from Keap1 and subsequently translocating into the cell nucleus (35, 36). In this study, it was confirmed that the elevating ROS activated $\mathrm{Nrf2}$, which then translocated into the nucleus, subsequently up-regulated its downstream target genes such as HO-1, NQO1, leading to inhibition of apoptosis, which is in consistent with the previous work $(31,37)$.

\section{REFERENCES}

1. Thrift AP, El-Serag HB. Burden of Gastric Cancer. Clin Gastroenterol Hepatol (2020) 18:534-42. doi: 10.1016/j.cgh.2019.07.045

2. Cao J, Zhang X, Xu P, Wang H, Wang S, Zhang L, et al. Circular RNA circLMO7 acts as a microRNA-30a-3p sponge to promote gastric cancer progression via the WNT2/beta-catenin pathway. J Exp Clin Cancer Res (2021) 40:6. doi: 10.1186/s13046-020-01791-9

3. Shen X, Wang J, Yan X, Ren X, Wang F, Chen X, et al. Predictive value of GSTP1 Ile105Val polymorphism in clinical outcomes of chemotherapy in

\section{CONCLUSION}

A novel Indole derivative LCT-3d was found to induce apoptosis in gastric cancer cells through DR5-mediated mitochondrial apoptotic pathway $(13,31)$. LCT-3d modulated the Bcl-2 family proteins and Cleaved-Caspases $3 / 9$, and resulted in cell apoptosis in MGC803 and HGC27 cells. Nrf2 played an important role in protecting gastric cancer cells from the injury of oxidative stress after LCT-3d treatment, but it could not reverse LCT-3d-induced cell apoptosis (Figure 7). This study suggested that LCT-3d could be a potential lead compound for the development of anti-gastric cancer agents.

\section{DATA AVAILABILITY STATEMENT}

The original contributions presented in the study are included in the article/supplementary material. Further inquiries can be directed to the corresponding authors.

\section{AUTHOR CONTRIBUTIONS}

MW, XW, LY, and Z-YH performed majority experiments, analyzed data, and wrote original draft. XL, XM, and C-TL synthesized the compound LCT-3d. G-YK and YC knocked down the DR5. C-YJ, H-WX, and ZW conceived and designed the experiments and revised the draft of manuscript. All authors contributed to the article and approved the submitted version.

\section{FUNDING}

The work was supported by the National Natural Science Foundation of China (Project No. 81973529 for C-YJ), "13th five-year plan of China": Major Projects of National Science and Technology on New Drug Creation and Development (Project No. HX2018ZX09711001-005-026 for HX), and Fang's family (Hong Kong) foundation (ZW).

\section{ACKNOWLEDGMENTS}

The author would like to thank Dr. Cheng-yun Jin, Dr. Hai-wei $\mathrm{Xu}$, and Dr. Zheng-ya Wang for providing technical support.

gastric and colorectal cancers: a systematic review and meta-analysis. Cancer Chemother Pharmacol (2016) 77:1285-302. doi: 10.1007/s00280-016-3047-1

4. Gao HL, Gupta P, Cui Q, Ashar YV, Wu ZX, Zeng L, et al. Sapitinib Reverses Anticancer Drug Resistance in Colon Cancer Cells Overexpressing the ABCB1 Transporter. Front Oncol (2020) 10:574861. doi: 10.3389/ fonc.2020.574861

5. Lei ZN, Teng QX, Zhang W, Fan YF, Wang JQ, Cai CY, et al. Establishment and Characterization of a Topotecan Resistant Non-small Cell Lung Cancer NCI-H460/TPT10 Cell Line. Front Cell Dev Biol (2020) 8:607275. doi: $10.3389 /$ fcell.2020.607275 
6. Wang JQ, Teng QX, Lei ZN, Ji N, Cui Q, Fu H, et al. Reversal of Cancer Multidrug Resistance (MDR) Mediated by ATP-Binding Cassette Transporter G2 (ABCG2) by AZ-628, a RAF Kinase Inhibitor. Front Cell Dev Biol (2020) 8:601400. doi: $10.3389 /$ fcell.2020.601400

7. Zhang H, Xu H, Ashby CJ, Assaraf YG, Chen ZS, Liu HM. Chemical molecular-based approach to overcome multidrug resistance in cancer by targeting P-glycoprotein (P-gp). Med Res Rev (2021) 41:525-55. doi: 10.1002/ med.21739

8. Munakarmi S, Chand L, Shin HB, Jang KY, Jeong YJ. Indole-3-Carbinol Derivative DIM Mitigates Carbon Tetrachloride-Induced Acute Liver Injury in Mice by Inhibiting Inflammatory Response, Apoptosis and Regulating Oxidative Stress. Int J Mol Sci (2020) 21:2048. doi: 10.3390/ijms21062048

9. Igarashi T, Kishi S, Hosono N, Higashi T, Iwao T, Yano R, et al. Population pharmacokinetic model development and exposure-response analysis of vincristine in patients with malignant lymphoma. Cancer Chemother Pharmacol (2021) 87. doi: 10.1007/s00280-020-04220-y

10. Lee CM, Park SH, Nam MJ. Anticarcinogenic effect of indole-3-carbinol (I3C) on human hepatocellular carcinoma SNU449 cells. Hum Exp Toxicol (2019) 38:136-47. doi: 10.1177/0960327118785235

11. Wang X, Wu E, Wu J, Wang TL, Hsieh HP, Liu X. An antimitotic and antivascular agent BPR0L075 overcomes multidrug resistance and induces mitotic catastrophe in paclitaxel-resistant ovarian cancer cells. PloS One (2013) 8:e65686. doi: 10.1371/journal.pone.0065686

12. Yang X, Wang W, Qin JJ, Wang MH, Sharma H, Buolamwini JK, et al. JKA97, a novel benzylidene analog of harmine, exerts anti-cancer effects by inducing G1 arrest, apoptosis, and p53-independent up-regulation of p21. PloS One (2012) 7:e34303. doi: 10.1371/journal.pone.0034303

13. Kim SY, Hwang S, Choi MK, Park S, Nam KY, Kim I. Molecular mechanisms underlying the effects of the small molecule AMC-04 on apoptosis: Roles of the activating transcription factor 4-C/EBP homologous protein-death receptor 5 pathway. Chem Biol Interact (2020) 332:109277. doi: 10.1016/ j.cbi.2020.109277

14. Yu H, Wu CL, Wang X, Ban Q, Quan C, Liu M, et al. SP600125 enhances C-2induced cell death by the switch from autophagy to apoptosis in bladder cancer cells. J Exp Clin Cancer Res (2019) 38:448. doi: 10.1186/s13046-019-1467-6

15. Jin CY, Molagoda I, Park C, Kwon TK, Yun SJ, Kim WJ, et al. PiceatannolInduced Apoptosis Is Reversed by N-Acetyl-L-cysteine through Restoration of XIAP Expression. Biol Pharm Bull (2018) 41:1372-8. doi: 10.1248/bpb.b1800157

16. Xu HW, Jia S, Liu M, Li X, Meng X, Wu X, et al. A low toxic CRM1 degrader: Synthesis and anti-proliferation on MGC803 and HGC27. Eur J Med Chem (2020) 206:112708. doi: 10.1016/j.ejmech.2020.112708

17. Kim SO, Cha HJ, Park C, Lee H, Hong SH, Jeong SJ, et al. Cordycepin induces apoptosis in human bladder cancer T24 cells through ROS-dependent inhibition of the PI3K/Akt signaling pathway. Biosci Trends (2019) 13:32433. doi: $10.5582 /$ bst.2019.01214

18. Naveen SM, Sivanandhan D, Gajendran C, Tantry S, Dewang P, Murugan K, et al. Novel dual LSD1/HDAC6 inhibitors for the treatment of multiple myeloma. Bioorg Med Chem Lett (2020) 34:127763. doi: 10.1016/ j.bmcl.2020.127763

19. Kim SY, Jin CY, Kim CH, Yoo YH, Choi SH, Kim GY, et al. Isorhamnetin alleviates lipopolysaccharide-induced inflammatory responses in BV2 microglia by inactivating NF-kappaB, blocking the TLR4 pathway and reducing ROS generation. Int J Mol Med (2019) 43:682-92. doi: 10.3892/ ijmm.2018.3993

20. Zingue S, Cisilotto J, Fogang R, Tchoupang EN, Ndinteh DT, Tchuenguem FN, et al. The antimammary tumor effects of ethanolic extract of propolis from Adamawa region (Cameroon) are by apoptosis via reactive oxygen species-mediated mitochondrial pathway. Environ Toxicol (2021) 36:861-73. doi: 10.1002/tox.23089

21. Wu Y, Xu J, Liu Y, Zeng Y, Wu G. A Review on Anti-Tumor Mechanisms of Coumarins. Front Oncol (2020) 10:592853. doi: 10.3389/fonc.2020.592853

22. Shakeri R, Kheirollahi A, Davoodi J. Apaf-1: Regulation and function in cell death. Biochimie (2017) 135:111-25. doi: 10.1016/j.biochi.2017.02.001
23. Jiang W, Chen Y, Li B, Gao S. DBA-induced caspase-3-dependent apoptosis occurs through mitochondrial translocation of cyt-c in the rat hippocampus. Mol Biosyst (2017) 13:1863-73. doi: 10.1039/c7mb00246g

24. Noh S, Choi E, Hwang CH, Jung JH, Kim SH, Kim B. Dietary Compounds for Targeting Prostate Cancer. Nutrients (2019) 11:11. doi: 10.3390/nu11102401

25. Jezek J, Cooper KF, Strich R. The Impact of Mitochondrial Fission-Stimulated ROS Production on Pro-Apoptotic Chemotherapy. Biol (Basel) (2021) 10:983. doi: 10.3390/biology10010033

26. Suraweera CD, Hinds MG, Kvansakul M. Poxviral Strategies to Overcome Host Cell Apoptosis. Pathogens (2020) 10:6. doi: 10.3390/pathogens10010006

27. Rachakhom W, Banjerdpongchai R. Effect of Calomelanone, a Dihydrochalcone Analogue, on Human Cancer Apoptosis/Regulated Cell Death in an In Vitro Model. BioMed Res Int (2020) 2020:4926821. doi: $10.1155 / 2020 / 4926821$

28. Negi A, Murphy PV. Development of Mcl-1 inhibitors for cancer therapy. Eur J Med Chem (2021) 210:113038. doi: 10.1016/j.ejmech.2020.113038

29. Quezada MJ, Picco ME, Villanueva MB, Castro MV, Barbero G, Fernandez NB, et al. BCL2L10 Is Overexpressed in Melanoma Downstream of STAT3 and Promotes Cisplatin and ABT-737 Resistance. Cancers (Basel) (2020) 13:78. doi: $10.3390 /$ cancers 13010078

30. Fu Y, Ye Y, Zhu G, Xu Y, Sun J, Wu H, et al. Resveratrol induces human colorectal cancer cell apoptosis by activating the mitochondrial pathway via increasing reactive oxygen species. Mol Med Rep (2021) 23:1. doi: 10.3892/ mmr.2020.11809

31. Zhang S, Li T, Zhang L, Wang X, Dong H, Li L, et al. A novel chalcone derivative S17 induces apoptosis through ROS dependent DR5 up-regulation in gastric cancer cells. Sci Rep (2017) 7:9873. doi: 10.1038/s41598-017-10400-3

32. Yogeswari S, Kamalraj S, Jayabaskaran C. A novel synergistic anticancer effect of fungal cholestanol glucoside and paclitaxel: Apoptosis induced by an intrinsic pathway through ROS generation in cervical cancer cell line (HeLa). Toxicol In Vitro (2021) 72:105079. doi: 10.1016/j.tiv.2021.105079

33. Wang YY, Lee KT, Lim MC, Choi JH. TRPV1 Antagonist DWP05195 Induces ER Stress-Dependent Apoptosis through the ROS-p38-CHOP Pathway in Human Ovarian Cancer Cells. Cancers (Basel) (2020) 12:1702. doi: 10.3390/ cancers 12061702

34. Cao X, Chen XM, Xiao WZ, Li B, Zhang B, Wu Q, et al. ROSmediated hypomethylation of PRDX5 promotes STAT3 binding and activates the Nrf2 signaling pathway in NSCLC. Int J Mol Med (2020) 47:573-82. doi: 10.3892/ ijmm.2020.4819

35. Kamble D, Mahajan M, Dhat R, Sitasawad S. Keap1-Nrf2 Pathway Regulates ALDH and Contributes to Radioresistance in Breast Cancer Stem Cells. Cells Basel (2021) 10:83. doi: 10.3390/cells10010083

36. Volpe C, Villar-Delfino PH, Dos AP, Nogueira-Machado JA. Cellular death, reactive oxygen species (ROS) and diabetic complications. Cell Death Dis (2018) 9:119. doi: 10.1038/s41419-017-0135-Z

37. Jin CY, Molagoda I, Karunarathne W, Kang SH, Park C, Kim GY, et al. TRAIL attenuates sulforaphane-mediated Nrf2 and sustains ROS generation, leading to apoptosis of TRAIL-resistant human bladder cancer cells. Toxicol Appl Pharmacol (2018) 352:132-41. doi: 10.1016/j.taap.2018.05.022

Conflict of Interest: The authors declare that the research was conducted in the absence of any commercial or financial relationships that could be construed as a potential conflict of interest.

The reviewer JH declared a shared affiliation with one of the authors, G-YK, to the handling editor at time of review.

Copyright (๑) 2021 Wang, Wu, Yu, Hu, Li, Meng, Lv, Kim, Choi, Wang, Xu and Jin. This is an open-access article distributed under the terms of the Creative Commons Attribution License (CC BY). The use, distribution or reproduction in other forums is permitted, provided the original author(s) and the copyright owner(s) are credited and that the original publication in this journal is cited, in accordance with accepted academic practice. No use, distribution or reproduction is permitted which does not comply with these terms. 